\begin{tabular}{|c|l|}
\hline Title & Levels of Transferrin in Bronchoalveolar Lavage Fluid in Sarcoidosis \\
\hline Author(s) & $\begin{array}{l}\text { Shigemura, Masahiko; Nasuhara, Y asuyuki; Konno, Satoshi; Hattori, Takeshi; Shimizu, Chikara; Matsuno, Kazuhiko; } \\
\text { Nishimura, Masaharu }\end{array}$ \\
\hline Citation & $\begin{array}{l}\text { Lung, 188(2), 151-157 } \\
\text { https://doi.org/10.1007/300408-009-9218-7 }\end{array}$ \\
\hline Issue Date & 2010-04 \\
\hline Doc URL & http://hdl.handle.net/2115/45089 \\
\hline Rights & The original publication is available at www.springerlink.com \\
\hline Type & article (author version) \\
\hline File Information & Lun1882_151-157.pdf \\
\hline
\end{tabular}

Instructions for use 


\section{Levels of Transferrin in Bronchoalveolar Lavage Fluid in}

\section{Sarcoidosis}

Masahiko Shigemura a, b Yasuyuki Nasuhara ${ }^{a^{*}}$ Satoshi Konno a Takeshi Hattori a Chikara Shimizu ${ }^{b}$ Kazuhiko Matsuno ${ }^{b}$ Masaharu Nishimura ${ }^{a}$

a Department of Respiratory Medicine, Graduate School of Medicine, Hokkaido University,

b Division of Laboratory and Transfusion Medicine, Hokkaido University Hospital, Sapporo, Japan

* Corresponding author. Department of Respiratory Medicine, Graduate School of Medicine, Hokkaido University, N-15 W-7, Kitaku, Sapporo, Japan.

Tel.: +81-11-716-1161 (Ext.5911), fax: +81-11-706-7899,

E-mail: nasuhara@med.hokudai.ac.jp (Y Nasuhara).

\section{Running title}

Transferrin in Sarcoidosis. 


\section{Abstract}

There has been only one report showing high levels of transferrin (Tf) in bronchoalveolar lavage fluid (BALF) in patients with sarcoidosis. The aim of this study is to assess the levels of Tf in both BALF and serum and to examine the relationship between the levels of $\mathrm{Tf}$ and other disease markers in sarcoidosis. Subjects were 64 sarcoidosis and 10 healthy controls. Tf in BALF and serum was measured by nephelometric assay. Median Tf levels in BALF from sarcoidosis was $0.70 \mathrm{mg} / \mathrm{dl}$ (range, 0.00-3.97), which was significantly higher when compared with controls ( $0.36 \mathrm{mg} / \mathrm{dl}$; range, $0.00-1.02)(\mathrm{p}=0.005)$. In contrast, median Tf levels in serum from sarcoidosis was $258 \mathrm{mg} / \mathrm{dl}$ (range, 171-383), which was significantly lower when compared with controls $(322 \mathrm{mg} / \mathrm{dl}$; range, 234-356) ( $\mathrm{p}=0.003)$. Tf levels in BALF were significantly correlated with both the percentage of lymphocytes ( $\mathrm{r}=0.617, \mathrm{p}=0.001)$ and serum angiotensin-converting enzyme activity $(r=0.363, p=0.003)$ and serum soluble interleukin-2 receptor $(r=0.450, p=0.001)$ in sarcoidosis. Levels of $\mathrm{Tf}$ in BALF from patients with sarcoidosis were not influenced by smoking status. The levels of Tf in sarcoidosis are high in BALF, but low in serum. Increased levels of Tf in BALF may reflect the disease activity.

Keywords Transferrin · Bronchoalveolar lavage fluid · Sarcoidosis. 


\section{Introduction}

Transferrin (Tf) is a major iron-binding glycoprotein with a molecular weight of $79500 \mathrm{Da}[1,2]$. Tf also plays essential roles in iron-binding and -transport, antimicrobial activity, cell growth, cell differentiation and cytoprotective processes [3, 4]. Tf is synthesized predominantly by hepatocytes, but is synthesized at lower levels in lymphocytes $[5,6]$. In the lung, Tf is also synthesized by pulmonary epithelial cells and submucosal glands [7]. As a percentage of total protein in bronchoalveolar lavage fluid (BALF), Tf levels are very high (4.0-5.6\%) when compared with values in plasma [2]. Tf is found not only in blood, but also in airway mucosa and alveolar lining fluids [2, 8]. Some reports have shown increased levels of BALF Tf in adult respiratory distress syndrome (ARDS) [9, 10] and decreased levels of BALF Tf in chronic obstructive pulmonary disease [10].

Sarcoidosis is a systemic granulomatous disorder of unknown etiology, and is characterized by accumulation of activated proliferating $\mathrm{T}$ lymphocytes in involved organs, most commonly the lungs. A typical feature of pulmonary sarcoidosis is an increase in the percentage of lymphocytes in BALF, with an accumulation of T-helper cells in the lung, resulting in a high proportion of CD4-positive lymphocytes in BALF [11-13]. On the other hand, Gallium-67 scanning for supporting the diagnosis of sarcoidosis is based on the uptake of the gallium-67 in granuloma, which is thought to be mediated by Tf receptors on alveolar marophages in alveorlar spaces and epitheliod cells in granuloma $[14,15]$. Expression of Tf receptors on alveolar macrophages is high in the granulomatous lung diseases [16]. Based on these, we hypothesize that Tf levels in BALF would be high in patients with sarcoidosis and relate to disease activity of sarcoidosis. To our knowledge, there has only been one report analyzing the relationship between Tf levels and cellular components in BALF from patients with sarcoidosis [17].

The aim of the present study is to simultaneously assess the levels of Tf in both BALF and serum from patients with sarcoidosis, and to further examine the relationship between the levels of $\mathrm{Tf}$ and other disease markers in sarcoidosis.

\section{Materials and Methods}

\section{Subjects}

Sixty-four patients (15 males and 49 females; median age, 54 years; range, 
18-75 years) were included based on a compatible clinical picture, histologic demonstration of noncaseating granulomas and exclusion of other diseases capable of producing a similar histrogic or clinical picture. Thirty patients were never smokers, 11 were former smokers and 22 were current smokers. The smoking status of 1 subject was unknown. The radiographical stage of sarcoidosis was stage 0 (no radiographic abnormalities) in 10 patients, stage I (bilateral hilar adenopathy without parenchymal abnormalities) in 40 patients, and stage II (bilateral hilar adenopathy with interstitial parenchymal infiltrate) in 14 patients. BAL findings and serum angiotensin-covering enzyme (ACE) activity are shown in Table 1. BALF cell analysis of three patients was not tested due to trouble in BAL storage. None of the patients received corticosteroid therapy prior to the BAL procedure.

Ten healthy volunteers ( 8 males and 2 females; median age, 22 years; range, 20-31 years), who were the students at our medical school with no history of any pulmonary disease or respiratory symptoms, served as control subjects. Five healthy volunteers were never smokers and 5 were current smokers.

There were significantly more females among the patients with sarcoidosis than in the healthy volunteers (chi-square test, $\mathrm{p}=0.001$ ) and the patients with sarcoidosis were significantly older than the healthy volunteers (Mann-Whitney U-test, $\mathrm{p}=0.001$ ).

All participants were Japanese and all gave written informed consent for participation in the study and all associated procedures. The Institutional Review Board of the School of Medicine, Hokkaido University, approved the study protocols.

\section{Blood analysis}

Venous blood samples were collected into Vacutainer ${ }^{\mathrm{TM}}$ tubes containing clot activator for serum preparations 30 minutes before BAL. The tubes were centrifuged at $3000 \mathrm{rpm}$ at room temperature for 10 minutes. Serum ACE activity was measured by colorimetric assay (ACE Color, Fujirebio Inc, Tokyo, Japan). Soluble interleukin 2 (IL-2) receptor was measured by a solid-phase, two-site chemiluminescent immunometric assay (IMMULITE 2000 IL2R, Siemens Healthcare Diagnostics, LA, USA). Serum samples for measuring Tf were immediately frozen and stored at $-80^{\circ} \mathrm{C}$ until assay.

\section{BAL}


Patients and healthy volunteers underwent BAL, as described elsewhere $[18,19]$. Briefly, the right middle lobe or the left counterpart of each subject was lavaged three times with $50-\mathrm{ml}$ aliquots (total volume: $150 \mathrm{ml}$ ) of sterile $0.9 \%$ saline at room temperature through a wedged flexible fiberoptic bronchoscope (Olympus 1T-200 or 1T-240, Tokyo, Japan). Recovered BAL fluid was separately filtered through several layers of gauze in order to remove excess mucus and debris, and was then centrifuged at $1500 \mathrm{rpm}$ for $5 \mathrm{~min}$ at $4^{\circ} \mathrm{C}$ in order to separate the supernatant from the cells. Aliquots of supernatant $(1 \mathrm{ml})$ were immediately frozen and stored at $-80^{\circ} \mathrm{C}$ prior to assay. Cell pellets were counted in a hematocytometer, and Diff-Quik ${ }^{\mathrm{TM}}$ (International Reagents, Kobe, Japan)-stained smears served to identify differential profiles after cytospin preparation.

Differential counts were performed by examining 300 cells using a standard light microscope. Flow cytometric analysis was performed right after the BAL procedure. All subjects refrained from smoking for at least 12 hours before the procedure in order to eliminate the acute effects of smoking.

\section{Measurement of Tf and albumin}

Levels of Tf in both BALF and serum were measured by nephelometric assay on a BN II Nephelometer (Dade Behring, Marburg, Germany). Levels of albumin in BALF were measured on a Hitachi 7070 automated analyzer with TAC-2 test Albumin U (Medical and biological laboratories co., LTD., Nagoya, Japan).

\section{Statistical methods}

Statistical analysis was performed with SPSS for Windows (SPSS Inc, USA) using non-parametric statistics. Comparisons were performed using the Mann-Whitney U-test. Differences between groups were evaluated by Kruskal-Wallis test. Correlations between different parameters were determined by Spearman's rank correlation coefficient. A p value of $<0.05$ was regarded as significant.

\section{Results}




\section{Levels of $\mathrm{Tf}$ in subjects with sarcoidosis and in healthy controls}

The median level of BALF Tf in subjects with sarcoidosis was $0.70 \mathrm{mg} / \mathrm{dl}$ (range, 0.00-3.97 mg/dl), which was significantly higher than in healthy controls (median, $0.36 \mathrm{mg} / \mathrm{dl}$; range, $0.00-1.02 \mathrm{mg} / \mathrm{dl})(\mathrm{p}=0.005)$ (Fig. 1A). In contrast, the median level of serum $\mathrm{Tf}$ in subjects with sarcoidosis was $258 \mathrm{mg} / \mathrm{dl}$ (range, 171-383 $\mathrm{mg} / \mathrm{dl}$ ), which was significantly lower than in healthy controls (median, $322 \mathrm{mg} / \mathrm{dl}$; range, 234-356 mg/dl) ( $\mathrm{p}=0.003$ ) (Fig. 1B). Next, the levels of Tf were analyzed for each radiographical stage. The median levels of BALF Tf in subjects at stage 0 , stage 1 and stage 2 were $0.86 \mathrm{mg} / \mathrm{dl}, 0.69 \mathrm{mg} / \mathrm{dl}$ and $0.72 \mathrm{mg} / \mathrm{dl}$, respectively. The median levels of serum Tf in the subjects at stage 0 , stage 1 and stage 2 were $265 \mathrm{mg} / \mathrm{dl}, 261 \mathrm{mg} / \mathrm{dl}$ and $245 \mathrm{mg} / \mathrm{dl}$, respectively. No statistically significant differences in levels of Tf were observed among the three stages in either BALF or in serum. In subjects with sarcoidosis, no significant differences were observed in the levels of BALF Tf between males and females ( $p=0.083$, NS, Fig. 2A) and no significant correlations were observed between age and levels of BALF Tf (r=-0.102, NS, Fig. 2B).

\section{Correlations between BALF Tf and BAL lymphocytes}

Levels of BALF Tf in sarcoidosis were significantly correlated with the percentage of lymphocytes $(\mathrm{r}=0.617 \mathrm{p}=0.001)$, the number of lymphocytes $(\mathrm{r}=0.494, \mathrm{p}=0.001)$ and the number of CD4 positive $\mathrm{T}$ cells $(\mathrm{r}=0.521, \mathrm{p}=0.001)$ in BALF, but there were no correlations in healthy controls (Fig. 3). There were no correlations between levels of Tf and CD4/CD8 ratio in BALF from subjects with sarcoidosis ( $\mathrm{r}=0.233, \mathrm{NS}$ ). No significant correlations were observed in the relationship between serum $\mathrm{Tf}$ and the percentage of lymphocytes $(\mathrm{r}=0.029, \mathrm{NS})$, the number of lymphocytes $(r=0.044, N S)$ and the number of CD4 positive $T$ lymphocytes ( $r=0.054, \mathrm{NS}$ ) in BALF from subjects with sarcoidosis.

\section{Correlations between Tf and serum ACE activity and serum soluble IL-2 receptor}

In sarcoidosis, significant correlations were observed between levels of BALF Tf and serum ACE activity ( $r=0.363, p=0.003$, Fig. 4 A) and serum soluble IL-2 receptor $(r=0.450, p=0.001$, Fig $5 \mathrm{~A}$ ), but there were no correlations between levels 
of serum Tf and serum ACE activity ( $r=-0.027$, NS, Fig. 4B) and serum soluble IL-2 receptor ( $r=-0.142$, NS, Fig 5B).

\section{Correlations between BALF Tf and BALF albumin}

A significant correlation was observed between the levels of Tf and albumin in BALF from subjects with sarcoidosis ( $r=0.943, p=0.001)$ (Fig. 6).

\section{Relationship between BALF Tf and smoking status}

We divided the patients with sarcoidosis into two groups of currently smokers and never smokers, and compared the cellular components in BALF with BALF Tf. The number of total cells in BALF from current smokers with sarcoidosis was $23.8 \times 10^{4} / \mathrm{ml}$ (range, 5.9-83.3 $\times 10^{4} / \mathrm{ml}$ ), which was significantly higher when compared to never smokers with sarcoidosis (median $13.6 \times 10^{4} / \mathrm{ml}$; range, $\left.5.1-32.5 \times 10^{4} / \mathrm{ml}\right)(\mathrm{p}<0.01)$. The percentage of lymphocytes in BALF from current smokers with sarcoidosis was $17.1 \%$ (range, 0.3-73.7\%), which was significantly lower when compared to never smokers with sarcoidosis (median $44.0 \%$; range, 11.7-76.3\%) $(\mathrm{p}<0.05)$. However, no statistically significant differences in the levels of BALF Tf were observed between current smokers (median, $0.57 \mathrm{mg} / \mathrm{dl}$; range, 0.00-3.02 $\mathrm{mg} / \mathrm{dl}$ ) and never smokers (median, 0.84 $\mathrm{mg} / \mathrm{dl}$; range, $0.25-3.97 \mathrm{mg} / \mathrm{dl}$ ). Thus, levels of BALF Tf in patients with sarcoidosis were not influenced by smoking status (Table 2).

\section{Discussion}

In the present study, we confirmed a previous report [17] demonstrating increased levels of BALF Tf in patients with sarcoidosis, as compared to healthy controls. In addition, we elucidated that levels of serum $\mathrm{Tf}$ in subjects with sarcoidosis were significantly lower than in healthy controls. In sarcoidosis, the levels of BALF Tf were significantly correlated with the percentage and number of lymphocytes in BALF, as well as with serum ACE activity and with serum soluble IL-2 receptor.

The percentage of lymphocytes in BALF is thought to be a marker of alveolitis [20,21]. Serum ACE in patients with sarcoidosis is considered to be produced by epithelioid cells, and the level of serum ACE activity reflects the total 
body granuloma load [22-24]. Soluble IL-2 receptor was reported to be helpful marker in predicting disease severity $[25,26]$. Therefore, BALF Tf might be one of the useful clinical markers in the assessment and follow-up of sarcoidosis.

Furthermore, measurements of Tf levels in BALF during exacerbation or before and after treatment would provide further important information on the clinical significance of Tf in sarcoidosis.

There are some other limitations in this study. There were significant differences in age and gender between the subjects with sarcoidosis and the healthy controls, with older subjects and more females being included among the sarcoidosis patients. However, no significant differences were observed in the levels of BALF Tf between females and males, and no significant correlations between age and BALF Tf levels were observed in sarcoidosis patients, thus suggesting that gender and age have little influence on BALF Tf values. To our knowledge, there have been no reports suggesting a possible influence of age or gender on Tf levels in BALF.

Smoking leads to a change in cellular components in BALF. Smoking increases the number of total cells and decreases the percentage of lymphocytes in BALF [21, 27]. However, it has been reported that levels of BALF Tf are not influenced by smoking status [2]. In the present study, no statistically significant differences were observed in the level of BALF Tf in sarcoidosis between current smokers and never smokers. Thus, BALF Tf does not appear to be influenced by smoking status in sarcoidosis.

The factors contributing to increased concentrations of Tf in BALF from patients with sarcoidosis are unclear. We speculate two mechanisms by which BALF Tf increases in sarcoidosis. One is that increased levels of BALF Tf are the result of an influx of plasma Tf into the alveoli. Previous reports have found that the increased levels of BALF Tf in ARDS result from an influx of plasma Tf into the alveoli as a result of increased disease activity $[9,10]$. In sarcoidosis, increased levels of BALF albumin are thought to result from an influx of plasma albumin into the alveoli as a result of increased disease activity [28]. To detect plasma leakage, we also measured the concentration of BALF albumin in subjects with sarcoidosis. A significant correlation was observed between the levels of Tf and albumin in BALF from subjects with sarcoidosis. The decrease in serum Tf observed in subjects with sarcoidosis may support this mechanism. The other possibility is that $\mathrm{Tf}$ is newly synthesized in the lung as sarcoidosis disease activity increases. Tf mRNA is known to be expressed in pulmonary epithelial cells and submucosal glands in the lung [7]. Tf has been reported to be present in human alveolar macrophages [29, 
30] and to be synthesized by lymphocytes [5, 6]. Therefore, numerous cell type may be capable of synthesizing Tf. A typical feature of pulmonary sarcoidosis is the increase in the percentage of lymphocytes in BALF with an accumulation of T-helper cells in the lung, thus resulting in a high proportion of CD4-positive lymphocytes in BALF [11-13]. Lum et al. reported the specific localization of Tf synthesis in CD4-positive lymphocytes [5]. On the other hand, IL-2, which plays a key role in T lymphocyte activation, is the most potent stimulator of Tf production in the human T-cell line Jurkat [6]. Pulmonary T lymphocytes in active pulmonary sarcoidosis also spontaneously release IL-2 [31]. Therefore, Tf might be locally synthesized in sarcoidosis, particularly by T lymphocytes in alveoli.

In summary, we assessed the levels of Tf in BALF and serum in patients with sarcoidosis, and we examined the relationship between the levels of $\mathrm{Tf}$ and other disease markers in sarcoidosis. The levels of Tf in sarcoidosis were high in BALF, but low in serum. Increased levels of Tf in BALF may reflect the disease activity. 


\section{References}

[1] Hirose M (2000) The structural mechanism for iron uptake and release by transferrins. Biosci Biotechnol Biochem 64:1328-1336

[2] Mateos F, Brock JH, Perez-Arellano JL (1998) Iron metabolism in the lower respiratory tract. Thorax 53:594-600

[3] Gomme PT, McCann KB (2005) Transferrin: structure, function and potential therapeutic actions. Drug Discov Today 10:267-273

[4] Dillner-Centerlind ML, Hammarstrom, Perlmann P (1979) Transferrin can replace serum for in vitro growth of mitogen-stimulated T lymphocytes. Eur J Immunol 9:942-948

[5] Lum JB, Infante AJ, Makker DM, Yang F, Bowman BH (1986) Transferrin synthesis by inducer T lymphocytes. J Clin Invest 77:841-849

[6]Djeha A, Pérez-Arellano JL, Hayes SL, Oria R, Simpson RJ, Raja KB, Brock JH (1995) Cytokine-mediated regulation of transferrin synthesis in mouse macrophages and human T lymphocytes. Blood 85:1036-1042

[7]Yang F, Friedrichs WE, Coalson JJ (1997) Regulation of transferrin gene expression during lung development and injury. Am J Physiol. 273:L417-L426

[8]Turi J, Yang F, Garrick MD, Piantadosi CA, Chio AJ (2004) The iron cycle and oxidative stress in the lung. Free Radic Biol Med 36:850-857

[9]Krsek-Staples JA, Kew RR, Webster RO (1992) Ceruloplasmin and transferrin levels are altered in serum and bronchoalveolar lavage fluid of patients with the adult respiratory distress syndrome. Am Rev Respir Dis 145:1009-1015

[10]Stites SW, Nelson ME, Wesselius LJ (1995) Transferrin concentrations in serum and lower respiratory tract fluid of mechanically ventilated patients with COPD or ARDS. Chest 107:1681-1685

[11]Statement on sarcoidosis (1999) Joint Statement of the American Thoracic 
Society (ATS), the European Respiratory Society (ERS) and the World Association of Sarcoidosis and Other Granulomatous Disorders (WASOG) adopted by the ATS Board of Directors and by the ERS Executive Committee, February 1999. Am J Resipr Crit Care Med 160:736-755

[12]Costabel U (2001) Sarcoidosis: clinical update. Eur Respir J 18(Suppl. 32): $56 s-68 s$

[13]Baughman RP, Lower EE, Bois RM (2003) Sarcoidosis. Lancet 361:1111-1118

[14]Sulavik SB, Spencer RP, Weed DA, Shapiro HR, Shiue ST, Castriotta RJ (1990) Recognition of distinctive patterns of gallium-67 distribution in sarcoidosis. J Nucl Med 31:1909-1914

[15]Tsuchiya Y, Nakao A, Komatsu T, Yamamoto M, Shimokata K (1992) Relationship between gallium 67 citrate scanning and transferrin receptor expression in lung diseases. Chest 102:530-534

[16]Haslam PL, Parker DJ, Townsend PJ (1990) Increases in HLA-DQ, DP, DR, and transferrin receptors on alveolar macrophages in sarcoidosis and allergic alveolitis compared with fibrosing alveolitis. Chest 97:651-661

[17]Popp W, Herkner K, Böck A, Rauscher H, Wanke T, Ritschka L, Zwick H (1992) Influences of the cellular and humoral immune system in bronchoalveolar lavage on lung function in pulmonary sarcoidosis. Respiration 59:89-93

[18]Yamaguchi E, Okazaki N, Tsuneta Y, Abe S, Terai T, Kawakami Y (1988) Interleukins in pulmonary sarcoidosis. Dissociative correlation of lung interleukins 1 and 2 with the intensity of alveolitis. Am Rev Respir Dis 138:645-651

[19]Zhou Y, Yamaguchi E, Fukui Y, Konno S, Maeda Y, Kimata K, Nishimura M (2005) Enhanced expression of interleukin-18 receptor alpha chain by CD4+ T cells in sarcoidosis. Chest 128:2497-2503

[20]No authors listed (1981) NIH conference. Pulmonary sarcoidosis: A disease characterized perpetuated by activated lung T lymphocytes. Ann Intern Med 
94:73-94

[21]Drent M, van Velzen-Blad H, Diamant M, Hoogsteden HC, van den Bosch JM (1993) Relationship between presentation of sarcoidosis and $T$ lymphocyte profile. A study in bronchoalveolar lavage fluid. Chest 104:795-780

[22]Silverstein E, Pertschuk LP, Friedland J (1979) Immunofluorescent localization of angiotensin converting enzyme in epithelioid and giant cells of sarcoidosis granulomas. Proc Natl Acad Sci USA 76:6646-6648

[23]Okabe T, Suzuki A, Ishikawa H, Yotsumoto H, Ohsawa N (1981) Cells originating from sarcoid granulomas in vitro. Am Rev Respir Dis 124:608-612

[24]Muthuswamy PP, Lopez-Majano V, Ranginwala M, Trainor WD (1987) Serum angiotensin-converting enzyme (SACE) activity as an indicator of total body granuloma load and prognosis in sarcoidosis. Sarcoidosis 4:142-148

[25]Ziegenhagen MW, Rothe ME, Schlaak M, Müller-Quernheim J (2003) Bronchoalveolar and serological parameters reflecting the severity of sarcoidosis. Eur Respir J 21:407-413

[26]Rothkrantz-Kos S, van Dieijen-Visser MP, Mulder PG, Drent M (2003) Potential usefulness of inflammatory markers to monitor respiratory functional impairment in sarcoidosis. Clin Chem 49:1510-1517

[27]Reynolds HY, Newball HH (1974) Analysis of proteins and respiratory cells obtained from human lungs by bronchial lavage. J Lab Clin Med 84:559-573

[28]Eklund A, Blaschke E (1986) Relationship between changed alveolar-capillary permeability and angiotensin converting enzyme activity in serum in sarcoidosis. Thorax 41:629-634

[29]Stecher VJ, Thorbecke GJ (1967) Sites of synthesis of serum proteins. 01. Serum proteins produced by macrophages in vitro. J Immunol 99:643-652

[30]Wesselius LJ, Flowers CH, Skikne BS (1992) Alveolar macrophage content of isoferritins and transferrin. Am Rev Respir Dis 145:311-316 
[31]Saltini C, Spurzem JR, Lee JJ, Pinkston P, Crystal RG (1986) Spontaneous release of interleukin 2 by lung $\mathrm{T}$ lymphocytes in active pulmonary sarcoidosis is primarily from the Leu3+DR+ T cell subset. J Clin Invest 77:1962-1970 


\section{Figure Legends}

\section{Fig. 1}

Levels of Tf in BALF and serum from patients with sarcoidosis and healthy controls. A, BALF Tf; B, serum Tf. The box represents the interquartile values $(25-75 \%)$ and the whiskers represent the non-outlying maximum and minimum. Bars in the box, median; circles, outliers.

\section{Fig. 2}

Influence of gender and age on levels of BALF Tf in sarcoidosis. A, Comparisons in levels of BALF Tf between males and females; B, Correlation between levels of BALF Tf and age. The box represents the interquartile values (25-75\%) and the whiskers represent the non-outlying maximum and minimum. Bars in the box, median; circles, outliers. No significant differences were observed in the levels of BALF Tf between females and males, and no significant correlation between age and levels of BALF Tf was observed in subjects with sarcoidosis.

\section{Fig. 3}

Correlations between levels of Tf and lymphocytes in BALF. Levels of BALF Tf in sarcoidosis were significantly correlated with the percentage of lymphocytes, the number of lymphocytes and the number of CD4 positive T cells in BALF, whereas there were no correlations in healthy controls.

\section{Fig. 4}

Correlations between levels of BALF Tf and serum ACE activity in patients with sarcoidosis. A, BALF Tf; B, serum Tf. In sarcoidosis, a significant correlation was observed between BALF Tf levels and serum ACE activity, whereas there was no correlation between levels of serum $\mathrm{Tf}$ and serum ACE activity.

\section{Fig. 5}

Correlations between levels of BALF Tf and serum soluble IL-2 receptor in patients with sarcoidosis. A, BALF Tf; B, serum Tf. In sarcoidosis, a significant correlation was observed between BALF Tf levels and serum soluble IL-2 receptor, whereas there was no correlation between levels of serum Tf and serum soluble IL-2 receptor.

\section{Fig. 6}


Correlations between BALF Tf and BALF albumin in patients with sarcoidosis. A significant correlation was observed between the levels of $\mathrm{Tf}$ and albumin in BALF from subjects with sarcoidosis. 
Healthy controls

Number of subjects

Male/ female

Age, yr

Cigarette smoking (never/former/current)

Radiographical stage (0/I/II)

BAL findings

Total cells, $\times 10^{4} / \mathrm{mL}$

Macrophages, \%

Lymphocytes, \%

CD4/CD8

Serum ACE activity, IU/L/37 $\mathrm{C}$

Data are presented as median (range).

${ }^{*} p<0.05,{ }^{* *} p<0.01$ vs. healthy controls

\section{0}

$8 / 2$

$22(20-31)$

$5 / 0 / 5$

$12.9(6.1-23.8)$

$88.9(70.2-97.4)$

$7.9(2.4-28.4)$

$1.32(0.47-3.00)$

\section{Sarcoidosis}

64

$15 / 49$ *

$54(18-75)$ *

$30 / 11 / 22$

$10 / 40 / 14$

17.4 (5.1-83.3) $69.2(22.3-96.8)$ ** $29.7(0.3-76.3)$ ** $4.43(0.36-15.48))^{* *}$ 23.0 (13.1-45.5)

\section{Table 1}




\section{Smoking status}

Currently smoking $(n=22)$ Never smoked $(n=30)$

BALF Tf, $\mathrm{mg} / \mathrm{dL}$

$0.57(0.00-3.02)$

$0.84(0.25-3.97)$

Total cells, $\times 10^{4} / \mathrm{mL}$

$23.8(5.9-83.3)$ **

$13.6(5.1-32.5)$

Lymphocytes, \%

$17.1(0.3-73.7)$ *

$44.0(11.7-76.3)$

Lymphocytes, $\times 10^{4} / \mathrm{mL}$

$3.8(0.0-39.7)$

$5.6(0.8-24.2)$

CD4 positive Tcells, $\times 10^{4} / \mathrm{mL}$

$3.1(0.0-29.0)$

$4.3(0.5-20.1)$

Data are presented as median (range).

${ }^{*} p<0.05,{ }^{* *} p<0.01$ vs. individuals who have never smoked 
A

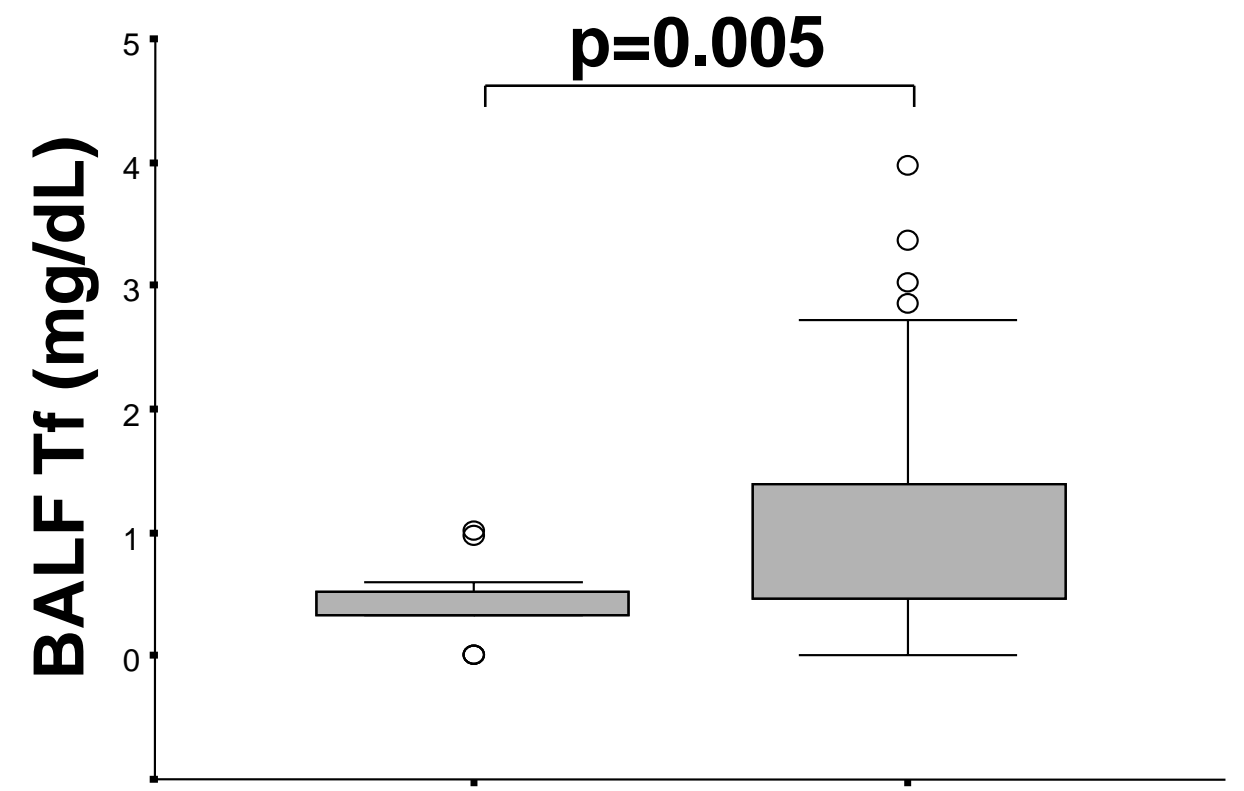

Healthy controls
B

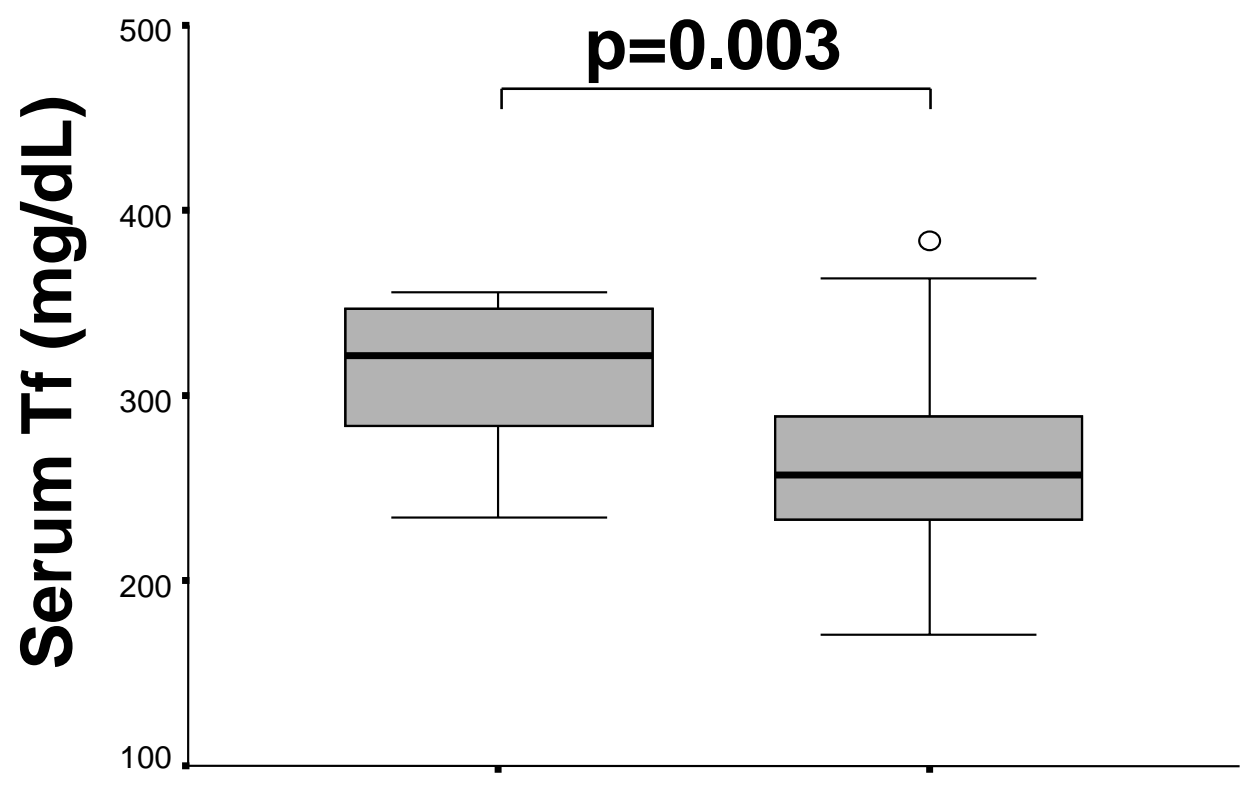

Healthy controls
Sarcoidosis 
A

B
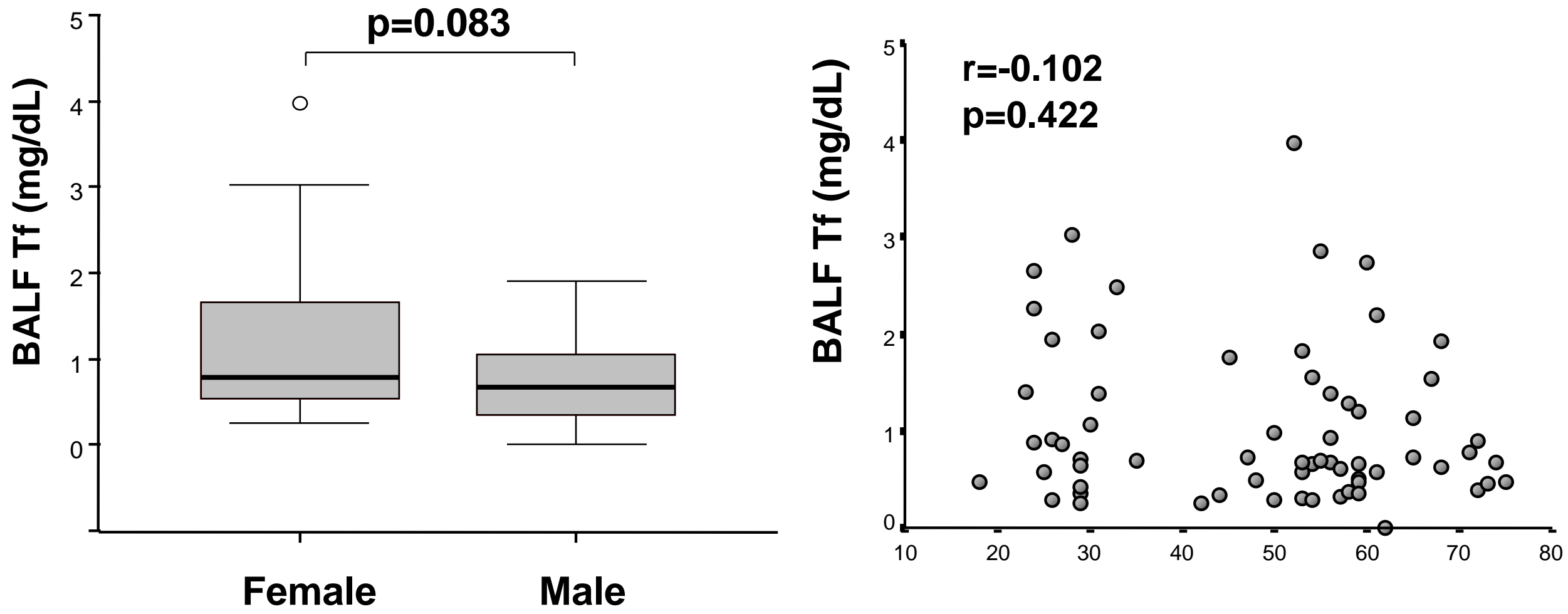

Age(yr)

Figure 2 
Sarcoidosis
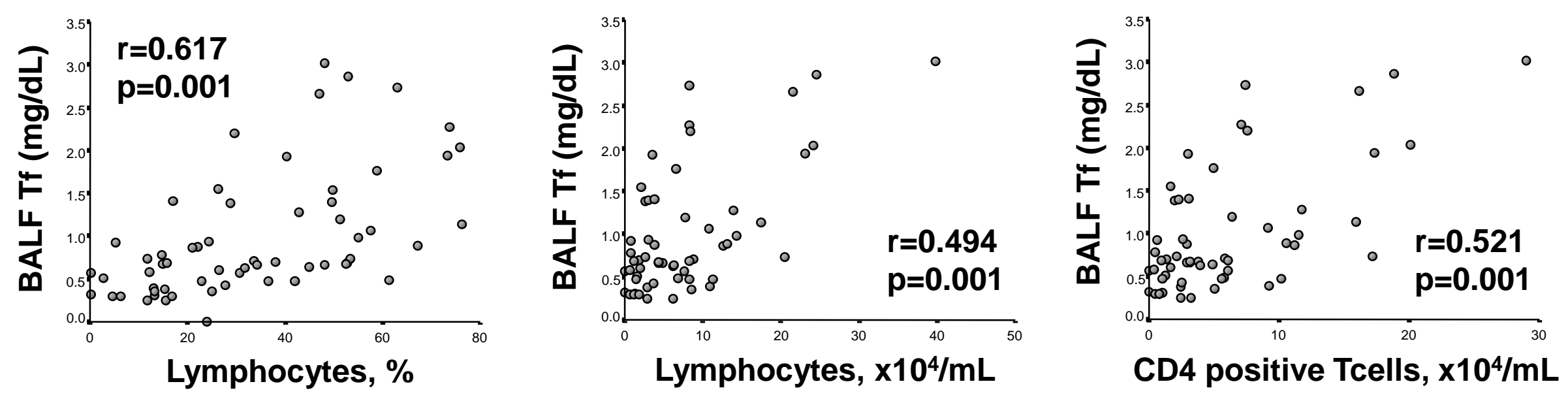

\section{Healthy controls}
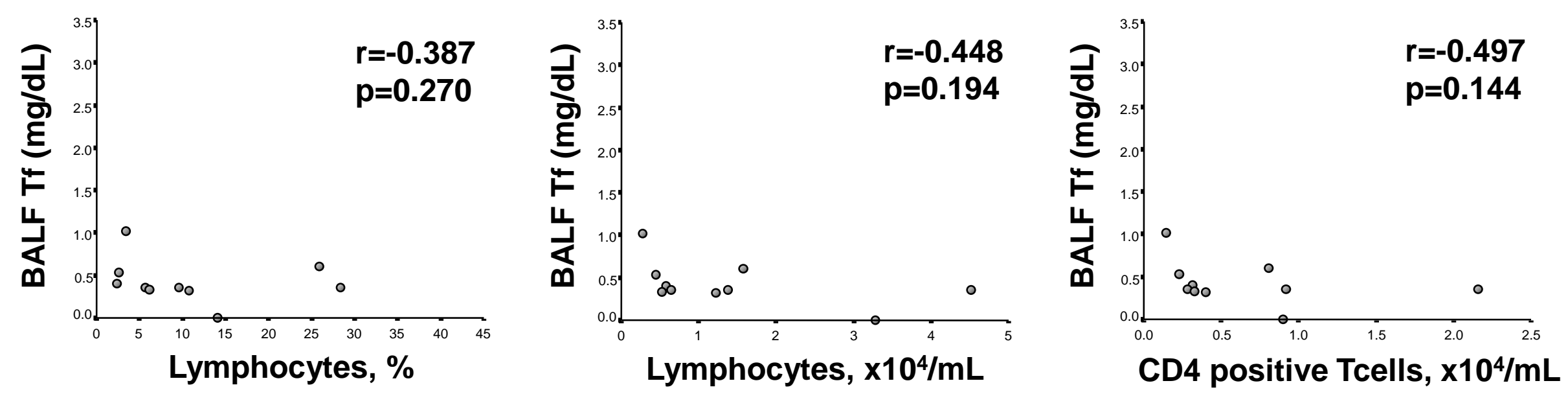

Figure 3 
A

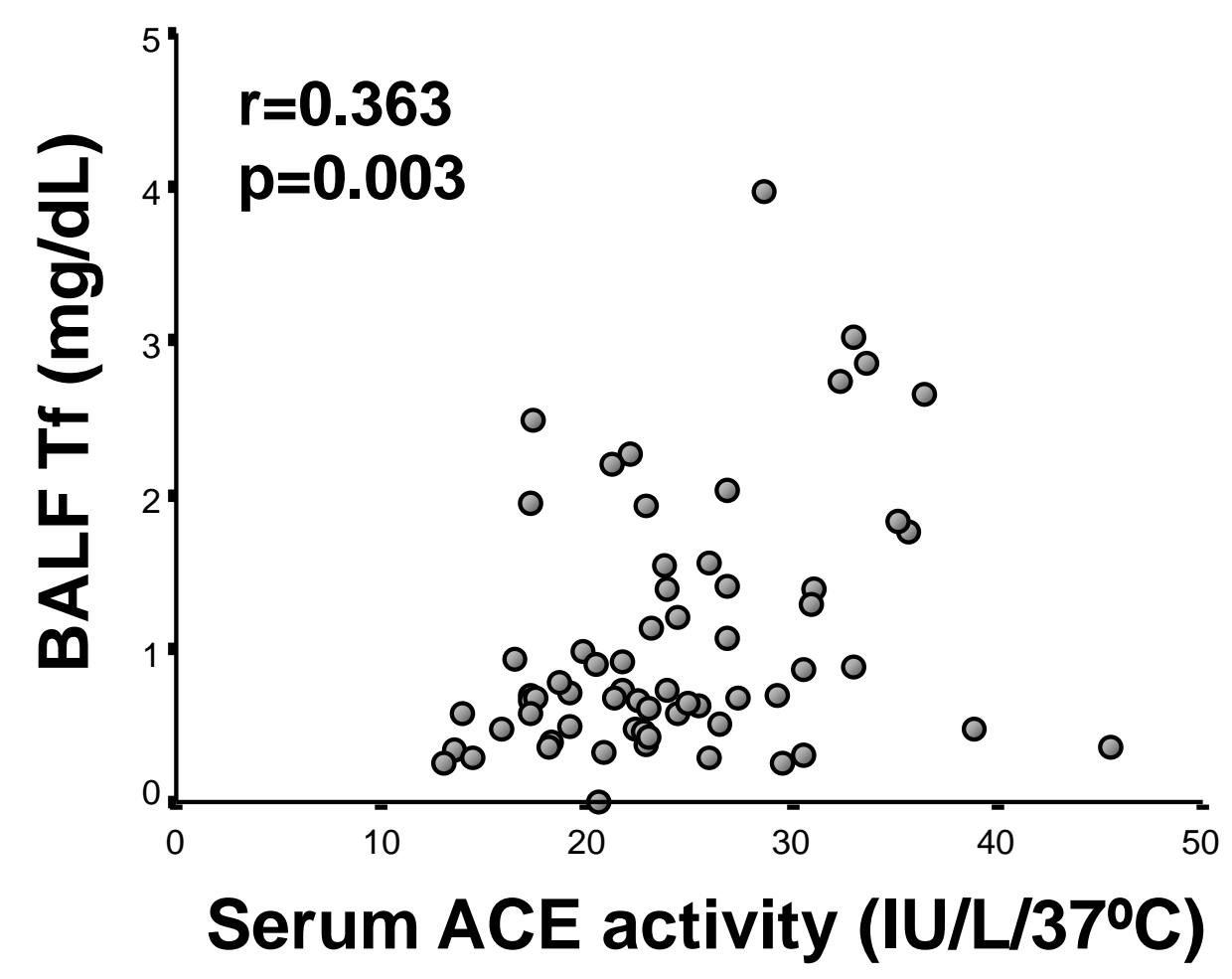

B

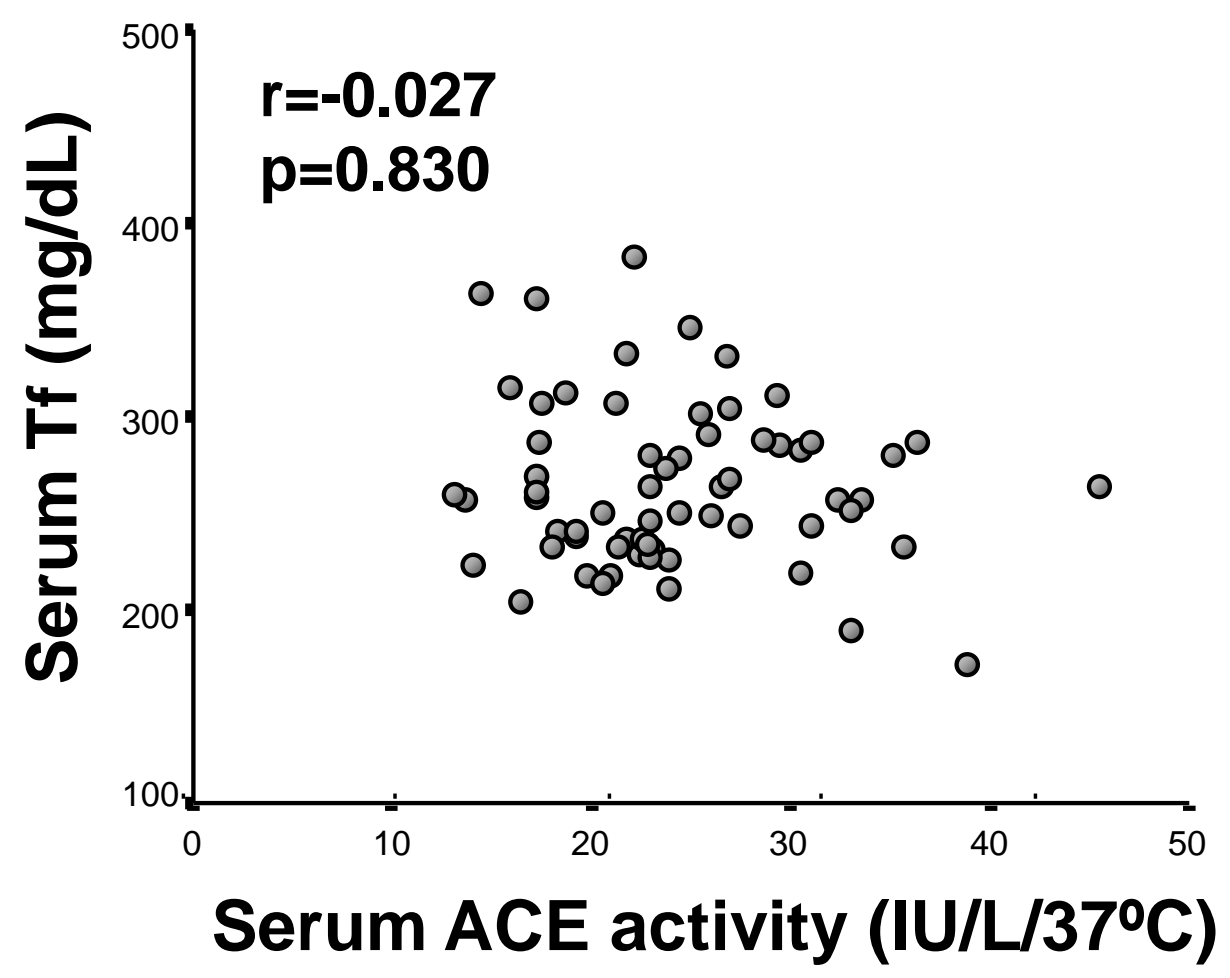

Figure 4 
A

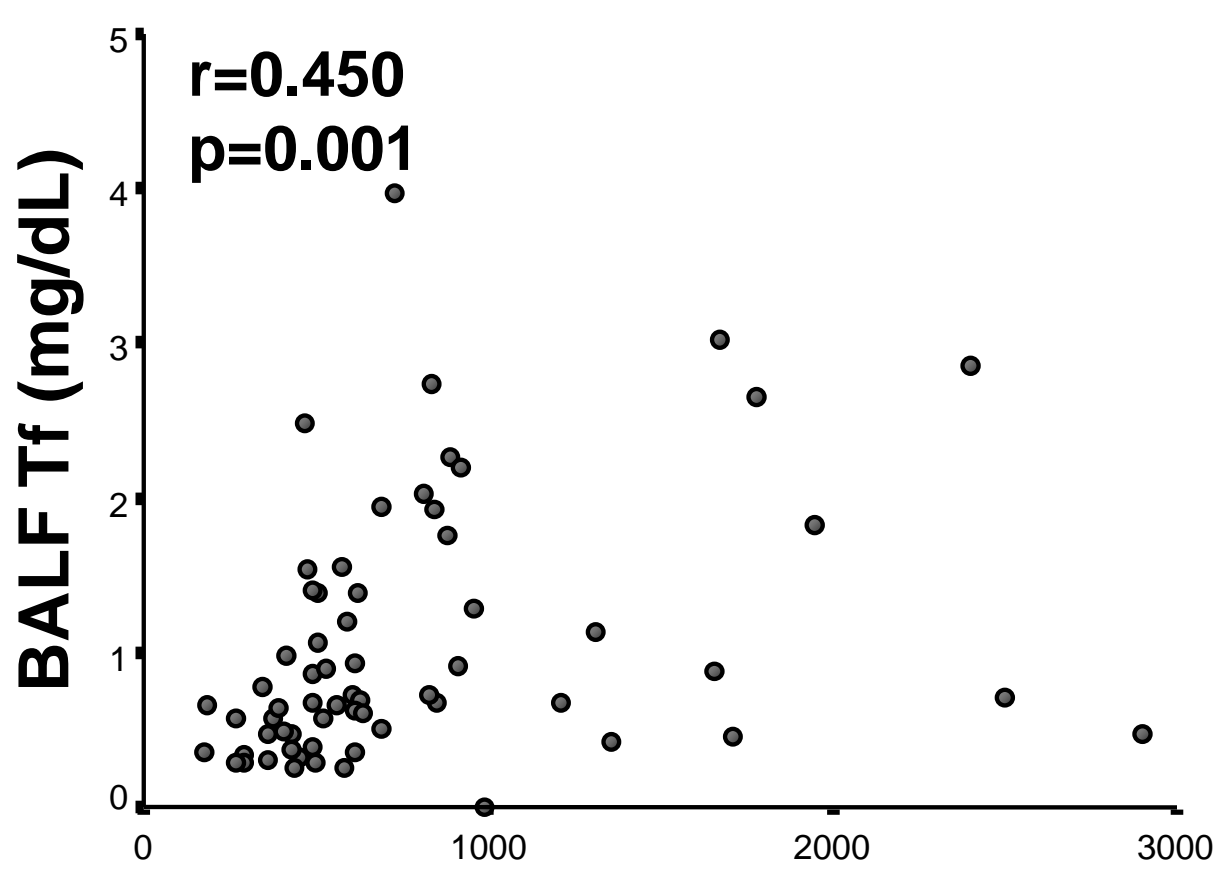

Serum soluble IL-2 receptor (U/mL)

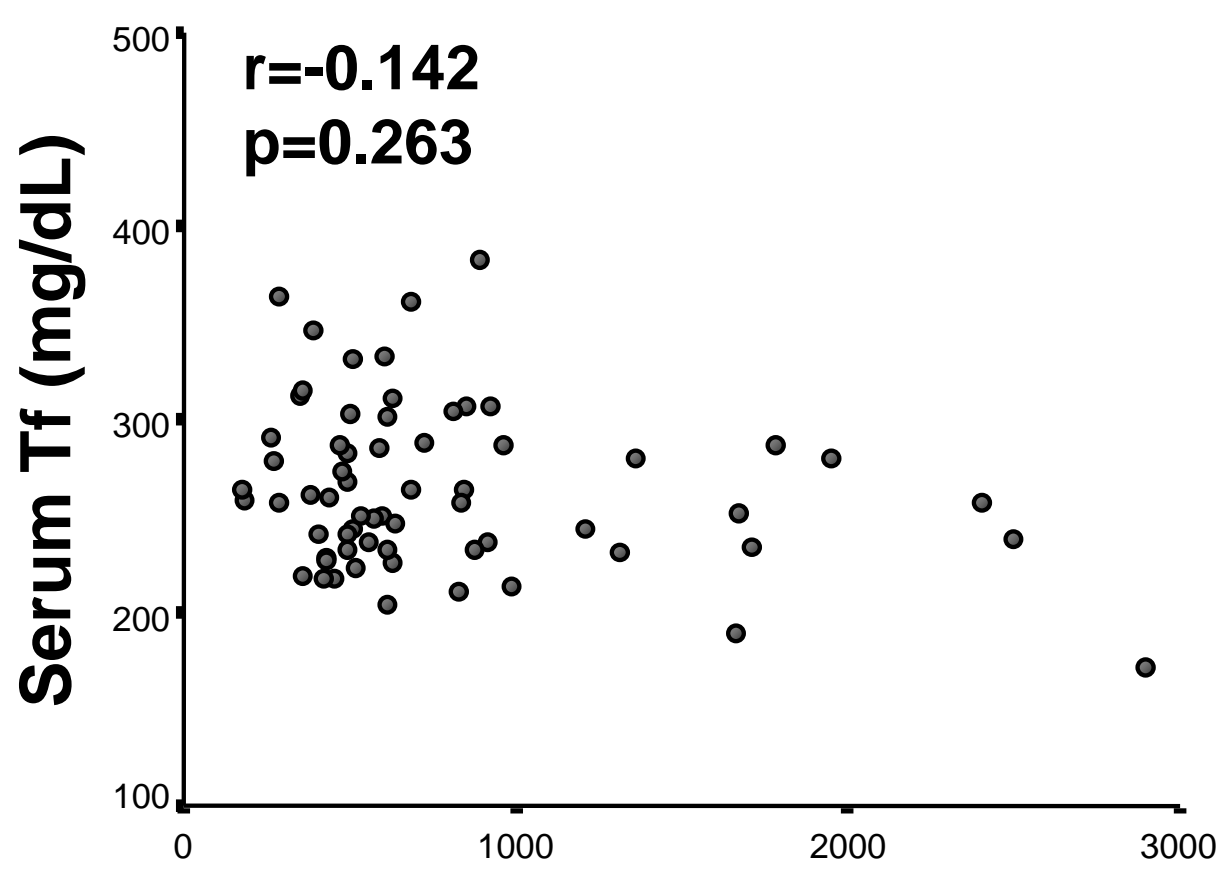

Serum soluble IL-2 receptor (U/mL)

Figure 5 


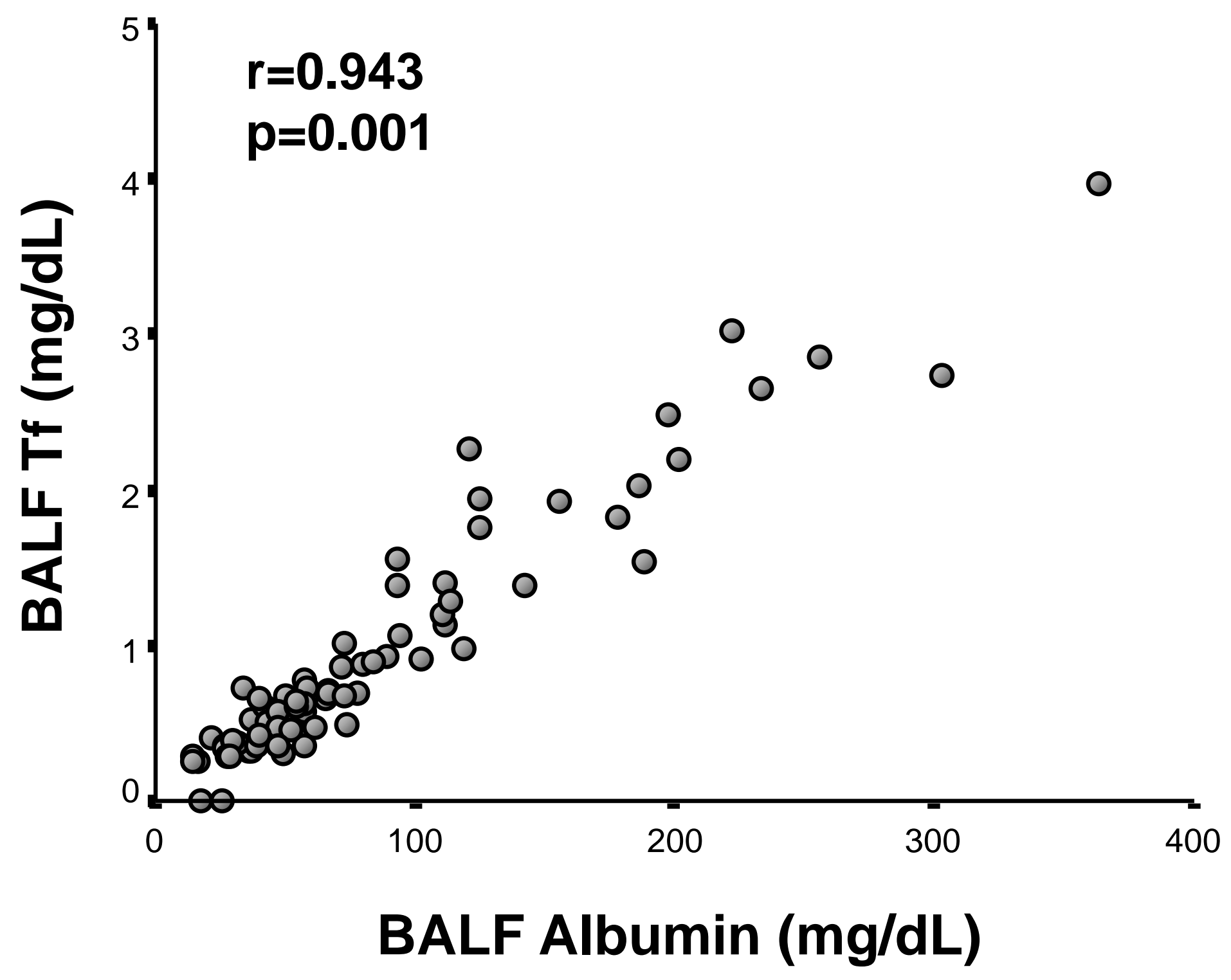

Figure 6 